\title{
Variação de atributos funcionais do componente arbóreo em função de gradientes edáficos em uma floresta nebular no sul do Brasil
}

Functional traits variation of the tree component in response to edaphic gradients in a cloud forest in the south of Brazil

\author{
Vanessa Fátima Soboleski ${ }^{1}$, Pedro Higuchi ${ }^{1,2}$, Ana Carolina da Silva ${ }^{1}$, Rodineli Loebens ${ }^{1}$, Karine Souza ${ }^{1}$, \\ Fernando Buzzi Junior ${ }^{1}$, Carla Luciane Lima ${ }^{1}$, Aline Pereira Cruz ${ }^{1}$, Mariele Alves Ferrer da Silva ${ }^{1}$ \\ \& Juliana Pizutti Dallabrida ${ }^{1}$
}

\begin{abstract}
Resumo
Objetivou-se investigar como a heterogeneidade ambiental influencia as variações espaciais dos atributos funcionais e a diversidade funcional do componente arbóreo em uma floresta nebular no sul do Brasil. Foram selecionadas as 19 espécies arbóreas mais abundantes identificadas em um inventário realizado em um fragmento florestal. As variáveis ambientais utilizadas foram obtidas de um trabalho prévio realizado na mesma área. Dessas espécies, foram obtidas a densidade da madeira, área foliar, área foliar específica, altura máxima potencial, deciduidade e síndrome de dispersão. Foram determinados os valores médios dos atributos ponderados para a comunidade e de diversidade funcional. Os dados ambientais foram ordenados por meio de Análise de Componentes Principais e modelos lineares foram ajustados relacionando os Componentes Principais significativos e os valores de atributos funcionais e de diversidade funcional. Enquanto os locais com maior fertilidade do solo favoreceram espécies com estratégias aquisitivas, representadas pela menor densidade da madeira e maior área foliar, e apresentaram maior diversidade funcional; os ambientes menos férteis favoreceram estratégias conservativas, representadas pela maior densidade da madeira e menor área foliar, e apresentaram uma menor diversidade funcional. Conclui-se que a floresta estudada apresentou o particionamento de habitats, em função de variações edáficas, por espécies com estratégias ecológicas distintas. Palavras-chave: diversidade funcional, estratégias ecológicas, variação ambiental.
\end{abstract}

\begin{abstract}
We aimed to investigate how the environmental heterogeneity affects the spatial variation of functional diversity and functional traits of a tree community in a cloud forest in the South of Brazil. For this, we selected the 19 most abundant species identified in a forest fragment survey. The study environmental variables were obtained from a previous study conducted in the same area. For each species, we measured the wood density, leaf area, specific leaf area and maximum potential height. We also determined the dispersal syndromes and deciduousness. We determined the community weighted mean for all traits and the functional diversity. The environmental data were ordered by Principal Component Analysis and Linear Models were fitted relating the significant Principal Components and the values of functional traits and of functional diversity. Whereas sites with higher fertility soils favored species with acquisitive strategies (i.e lower wood density and greater leaf area), and showed a higher functional diversity; lower fertility soils sites favored conservative strategies (i.e higher wood density and smaller leaf area), and had a lower functional diversity. We conclude that the studied forest showed habitat partitioning by species with different ecological strategies, in function of edaphic variations.
\end{abstract}

Key words: functional diversity, ecological strategies, environmental variation. 


\section{Introdução}

O entendimento dos processos que influenciam a organização de comunidades de espécies arbóreas em ambientes naturais tem sido um dos principais focos na área de ecologia vegetal, uma vez que esta é uma questão-chave para a definição de estratégias adequadas de manejo e restauração florestal. Neste sentido, vários estudos no Brasil têm adotado uma abordagem com enfoque taxonômico, sem considerar aspectos funcionais, demonstrando a influência de fatores ecológicos, como condições ambientais (Torres et al. 1997; Oliveira-Filho \& Fontes 2000; Moro et al. 2015), competição inter e intraespecífica (Martins et al. 2004) e outros processos ecológicos espacialmente estruturados (Fernandes \& Queiroz 2015; Maçaneiro et al. 2016), sobre a distribuição de espécies arbóreas em ecossistemas florestais. Porém, mais recentemente, estudos começaram a empregar abordagens nas quais as espécies são tratadas como entidades funcionalmente distintas (Magnago et al. 2014; Rosado et al. 2016), permitindo inferências ecológicas com um viés evolutivo, em que algumas características morfológicas, ecológicas e fisiológicas são consideradas como atributos funcionais e podem indicar a aptidão de uma espécie para se desenvolver em uma determinada condição ambiental (Violle et al. 2007).

As características funcionais influenciam a capacidade de sobrevivência dos organismos e resumem as inúmeras formas que estes se beneficiam de um determinado recurso para o seu desenvolvimento (Violle et al. 2007). De forma geral, no que se refere às estratégias ecológicas, as espécies de plantas têm sido agrupadas em dois grupos: aquelas com estratégias aquisitivas, que desenvolveram em características que otimizam/ maximizam a captura de recursos, e aquelas com estratégias conservativas, que investem em mecanismos de proteção da biomassa, de forma a conservar os recursos adquiridos (Díaz et al. 2004; Reich 2014). Os atributos funcionais das espécies são representados por algumas características chave (Violle \& Jiang 2009), como, por exemplo, a área foliar, a densidade básica da madeira e a altura máxima (Kraft et al. 2008). Geralmente, as estratégias aquistivas podem ser expressas funcionalmente por meio de uma maior taxa de crescimento, menor densidade da madeira, maior área foliar e maior área foliar específica, por outro lado, as estratégias conservativas podem refletir em características funcionais antagônicas (Reich 2014). Enquanto estratégias aquisitivas proporcionam vantagens em ambientes com maior disponibilidade de recurso, estratégias conservativas oferecem vantagens quando os recursos são escassos (ApazaQuevedo et al. 2015). Assim, espera-se que em escala local ( $<1$ ha) de ecossistemas florestais sejam observadas variações dos atributos funcionais em reposta a gradientes ambientais, como as variações no relevo e nas propriedades físico-químicas dos solos, que influenciam a disponibilidade de nutrientes, refletindo no particionamento de habitats por diferentes espécies. Ou seja, espécies distintas ocupariam diferentes porções de gradientes ambientais, em função de suas respectivas aptidões para tais condições (fitness). Neste sentido, um questionamento que emerge diante da base teórica já existente é se este padrão pode ser generalizado para florestas condicionadas pela existência de fortes filtros ambientais, como, por exemplo, nas florestas nebulares no sul do Brasil, que apresentam baixas temperaturas, geadas frequentes e nebulosidade constante (Falkenberg \& Voltolini 1995), com ocorrência de neve no inverno.

As florestas altomontanas, além de condições ambientais específicas e restritivas para muitas espécies florestais, apresentam uma grande importância ecológica, pois possuem um elevado endemismo de espécies (Falkenberg \& Voltolini 1995; Safford 1999; Safford 2007; Scheer \& Mocochinski 2009) e desempenham importantes funções ambientais (e.g., manutenção do ciclo hidrológico e de um elevado depósito de carbono no solo) (Kitayama \& Aiba 2002; Martínez et al. 2009; Scheer et al. 2011a). Porém, este ecossistema encontra-se, atualmente, ameaçado devido ao histórico de fragmentação florestal, perturbações antrópicas crônicas e possíveis processos de mudanças climáticas (Becker et al. 2007).

Vários estudos (Falkenberg \& Voltolini 1995; Becker et al. 2007; Homeier et al. 2010; Scheer et al. 2011b; Higuchi et al. 2013; Marcon et al. 2014) têm demonstrado que as florestas nebulares, além de uma baixa diversidade, apresentam características fisionômicas peculiares, como a presença de um único estrato vertical, constituído por árvores de pequeno porte, com troncos tortuosos, folhas pequenas e coriáceas e cobertas por epífitas. Estas características representam adaptações aos filtros ambientais existentes, como a baixa disponibilidade de nutrientes nos solos, a influência do vento, a temperatura reduzida e a presença constante de nuvens, que resultam em menores taxas de decomposição da serapilheira, absorção de nutrientes e produtividade primária (Leigh 1975; Homeier et al. 2010; Scheer et al. 2011b). 
Desta forma, considerando a importância de pesquisas ecológicas em áreas de floresta nebular, o presente estudo objetivou avaliar como a heterogeneidade ambiental (propriedades físicoquímicas do solo, relevo, cobertura do dossel e impacto ambiental) exerce influência sobre a variação espacial dos atributos funcionais e da diversidade funcional em uma comunidade de espécies arbóreas de uma floresta nebular no sul do Brasil. Tendo em vista a heterogeneidade do ambiente edáfico na área, que foi observado por Marcon et al. (2014) em estudo prévio, associado à topografia e à disponibilidade de nutrientes, testou-se a hipótese de que existe o particionamento espacial por espécies com diferentes atributos funcionais, com locais com solos de maior fertilidade natural favorecendo estratégias aquisitivas, e locais com solos menos férteis, favorecendo estratégias conservativas.

\section{Material e Métodos}

O fragmento de floresta nebular estudado, segundo Marcon et al. (2014), possui área de 346 ha e está localizada no município de Urubici, no Planalto Catarinense, sul do Brasil, na latitude 28 $04^{\prime} 27^{\prime}$ 'S e longitude 49 37'30'O. Apresenta uma altitude aproximada de $1.600 \mathrm{~m}$, com ocorrência de geada e neve no inverno. A região possui clima $\mathrm{Cfb}$, temperado com verões amenos, de acordo com a classificação de Köppen, com temperatura média anual que varia de 11 a $15^{\circ} \mathrm{C}$ (Pandolfo et al. 2002). A precipitação média anual varia entre 1.200 e $1.900 \mathrm{~mm}$, com chuvas bem distribuídas durante $\mathrm{o}$ ano. Os solos da região são pouco profundos e classificados, principalmente, como Neossolos Litólicos e Cambissolos. Segundo a classificação do IBGE (2012), a vegetação arbórea é descrita como Floresta Ombrófila Mista Alto-Montana.

Para a caracterização dos atributos funcionais das espécies arbóreas, foram consideradas as 19 espécies lenhosas mais abundantes no fragmento estudado, amostradas previamente por Marcon et al. (2014), a partir do levantamento florístico-estrutural das espécies arbóreas em 25 parcelas de $400 \mathrm{~m}^{2}$ cada $(20 \times 20 \mathrm{~m})$, alocadas de forma sistemática, distanciadas $10 \mathrm{~m}$ entre si. Entre as mais abundantes, somente Dicksonia sellowiana Hook. foi retirada, pois não é uma espécie lenhosa, o que iria inviabilizar a obtenção de alguns atributos funcionais. As 19 espécies selecionadas somavam $57,58 \%$ das espécies e $91,07 \%$ dos indivíduos previamente amostrados nas parcelas, representando, assim, a maior parte da florística e estrutura da floresta amostrada, sendo um percentual de abundância maior do que o recomendado por Pakeman \& Quested (2007), que é de $80 \%$ para a caracterização de atributos funcionais. Nessas mesmas parcelas utilizadas para o levantamento florístico-estrutural, Marcon et al. (2014) também realizaram a caracterização ambiental: características edáficas de $\mathrm{pH}$, fósforo $(\mathrm{P})$, potássio $(\mathrm{K})$, cálcio $(\mathrm{Ca})$, magnésio $(\mathrm{Mg})$, sódio $(\mathrm{Na})$, alumínio ( $\mathrm{Al})$, matéria orgânica $(\mathrm{MO})$, capacidade de troca catiônica (CTC), saturação por base $(\mathrm{V})$, teores de areia, silte, argila e compactação do solo (resistência à penetração a $20 \mathrm{~cm}$ de profundidade no solo e resistência do valor máximo no perfil), caracterização topográfica (cota, declividade média e desnível máximo), cobertura do dossel e impacto ambiental. As propriedades físico-químicas dos solos foram obtidas por meio de amostras compostas em cada parcela, oriundas de nove coletas realizadas na camada superficial de 0 a $20 \mathrm{~cm}$. Neste estudo, Marcon et al. (2014) observaram elevada heterogeneidade edáfica, associada a topografia e fertilidade do solo, com locais mais planos apresentando solos de baixa fertilidade natural e locais mais declivosos, solos de maior disponibilidade de nutrientes.

No presente estudo, para cada uma das 19 espécies, foram caracterizados os atributos funcionais de densidade básica da madeira (g/ $\left.\mathrm{cm}^{3}\right)$, área foliar $\left(\mathrm{cm}^{2}\right)$, área foliar específica $\left(\mathrm{cm}^{2} / \mathrm{g}\right)$, altura máxima potencial $(\mathrm{m})$, guilda de dispersão (zoocórica, anemocórica ou autocórica) e deciduidade (decídua, semi-decídua e perene). Para determinação da densidade básica da madeira, utilizou-se o protocolo de Chave (2005), sendo coletadas amostras de madeira de, no mínimo, cinco indivíduos de cada espécie presente no fragmento estudado, com DAP de 10 a $30 \mathrm{~cm}$, por meio de um trado de incremento (diâmetro de 5,15 mm). As amostras foram condicionadas em recipientes com água até que apresentassem massa úmida constante. $\mathrm{O}$ volume da amostra foi determinado pelo princípio de Arquimedes, onde a medida da massa de água deslocada é igual ao volume da amostra. Posteriormente, as amostras foram colocadas em estufa, com temperatura de $67^{\circ} \mathrm{C}$, até que a massa seca permanecesse constante e então pesadas em balança analítica com precisão de 0,001 g. Dessa maneira, a razão entre a massa seca $(\mathrm{g})$ e o volume $\left(\mathrm{cm}^{3}\right)$ gerou a densidade básica da madeira $\left(\mathrm{g} . \mathrm{cm}^{-3}\right)$, obtendo-se, posteriormente, a média da densidade básica dos indivíduos de cada espécie.

A área foliar e a área foliar específica foram obtidas por meio da coleta de 20 folhas, de 10 indivíduos por espécie, localizados no fragmento, incluindo os pecíolos e, para folhas compostas, 
raques, seguindo o protocolo de Pérez-Harguindeguy et al. (2013). Foram coletadas folhas do terço mediano da copa, dos indivíduos que obtivessem maior incidência de luz e as mais sadias, preferencialmente. Em seguida, as folhas foram "escaneadas" e as imagens processadas no programa ImageJ (Rasband 2007), onde foram obtidas as áreas foliares $\left(\mathrm{cm}^{2}\right)$, calculando-se, posteriormente, a média das áreas foliares por espécie. Em seguida, as folhas de todos os indivíduos de cada espécie foram armazenadas em estufa até que alcançaram a massa seca constante e pesadas juntas em balança analítica com precisão de $0,001 \mathrm{~g}$. Optou-se por pesar todas as folhas da mesma espécie juntas para evitar-se imprecisão da balança em relação àquelas espécies de folhas pequenas. A área foliar específica $\left(\mathrm{cm}^{2} \cdot \mathrm{g}^{-1}\right)$ foi obtida pela a razão entre a área foliar $\left(\mathrm{cm}^{2}\right)$ e a massa seca das folhas $(\mathrm{g})$.

A altura máxima potencial de cada espécie, representando o tamanho das árvores em suas idades adultas no fragmento estudado, foi caracterizada a partir das alturas estimadas por Marcon et al. (2014) para os indivíduos amostrados das espécies avaliadas, que, posteriormente, foram comparadas com os valores observados na literatura (Lorenzi 2002, 2008, 2009). As guildas de dispersão das espécies foram avaliadas de acordo com a classificação proposta por Van Der Pijl (1982), classificando as espécies em zoocóricas, anemocóricas ou autocóricas, a partir de observações no campo quanto às características de fruto e revisão de literatura (e.g., Negrini et al. 2012; Souza et al. 2015). A deciduidade das espécies foi avaliada por meio de observações a campo e revisão de literatura (Lorenzi 2002, 2008). As observações de campo foram baseadas na classificação fitofisionômica proposta por IBGE (2012), de forma que espécies que apresentaram perda de até $20 \%$ de suas folhas no período desfavorável foram classificadas como perenes, espécies que perderam de 20 a $50 \%$ das folhas foram classificadas como semi-decíduas e espécies com uma perda de folhas maior do que 50\% foram classificadas como decíduas.

Para cada parcela, foram determinados os valores médios dos atributos funcionais ponderados para a comunidade (CWM) das 19 espécies estudadas e a diversidade funcional, por meio do índice de RaoQ. Os valores de CWM representam os valores de atributos funcionais mais prováveis de serem observados para uma determinada espécie, a partir de um indivíduo selecionado ao acaso (Garnier \& Navas 2012). O índice de RaoQ foi proposto por BottaDukát (2005), baseado na entropia quadrática de Rao (1982), sendo considerado um índice eficiente para a caracterização da diversidade funcional, uma vez que considera a abundância de indivíduos e mais do que um único atributo funcional (Botta-Dukát 2005).

Os gradientes ambientais, determinados a partir das variáveis ambientais das parcelas, foram identificados por meio da Análise de Componentes Principais (PCA), junto com a análise de Scree Plot, para identificar os eixos da PCA que explicaram um percentual significativo da variação total dos dados. A influência de gradientes ambientais, representados pelos eixos da PCA, sobre os atributos funcionais (CWM) e diversidade funcional (RaoQ) determinados para as parcelas, foi examinada por meio de modelos lineares simples, cujos resíduos foram avaliados quanto à sua distribuição normal, por meio do teste de Shapiro-Wilk, quanto à sua a homocedasticidade, por meio da análise gráfica, e quanto à sua dependência espacial, por meio de correlograma de Moran. Como em todas as parcelas ocorreu a dominância de espécies zoocóricas e perenifólias, não existindo variação em relação a estes atributos, os mesmos não foram considerados na modelagem. Todas as análises foram realizadas no ambiente de programação estatística $R$ ( $R$ Development Core Team 2015), utilizando-se os pacotes vegan (Oksanen et al. 2015), FD (Laliberté \& Shipley 2014) e ncf (Bjornstad 2013).

\section{Resultados}

\section{Gradientes ambientais}

Os dois primeiros eixos da PCA explicaram $61,20 \%$ da variação total, com os eixos 1 e 2 explicando 46,59\% e 14,60\%, respectivamente. De acordo com a análise de Scree Plot, ambos os eixos explicaram uma proporção significativa da variação total, uma vez que a inércia observada (círculos fechados e linha contínua) foi maior do que um valor esperado aleatório (círculos abertos e linha pontilhada), indicado pela distribuição de Broken Stick (Fig. 1a).

As variáveis ambientais de maiores correlações com o eixo 1 da PCA foram $\mathrm{Al}(-0,30), \mathrm{pH}$ em água $(0,30), \mathrm{Ca}(0,29)$, saturação por base $(0,28)$, areia $(-0,28)$, matéria orgânica $(0,27), \mathrm{CTC}$ $(-0,26)$ e declividade $(0,26)$. Já as variáveis mais correlacionadas com o eixo 2 foram resistência a penetração no solo a $20 \mathrm{~cm}$ de profundidade $(-0,48)$, $\mathrm{K}(-0,34), \operatorname{Mg}(-0,27)$, cobertura do dossel $(-0,25)$ e cota média $(0,24)$. Desta forma, pode-se identificar, ao longo do eixo 1, um gradiente edáfico associado, principalmente, à acidez, fertilidade, textura do solo e declividade; e, ao longo do eixo 2, um gradiente ambiental associado, principalmente, à compactação 

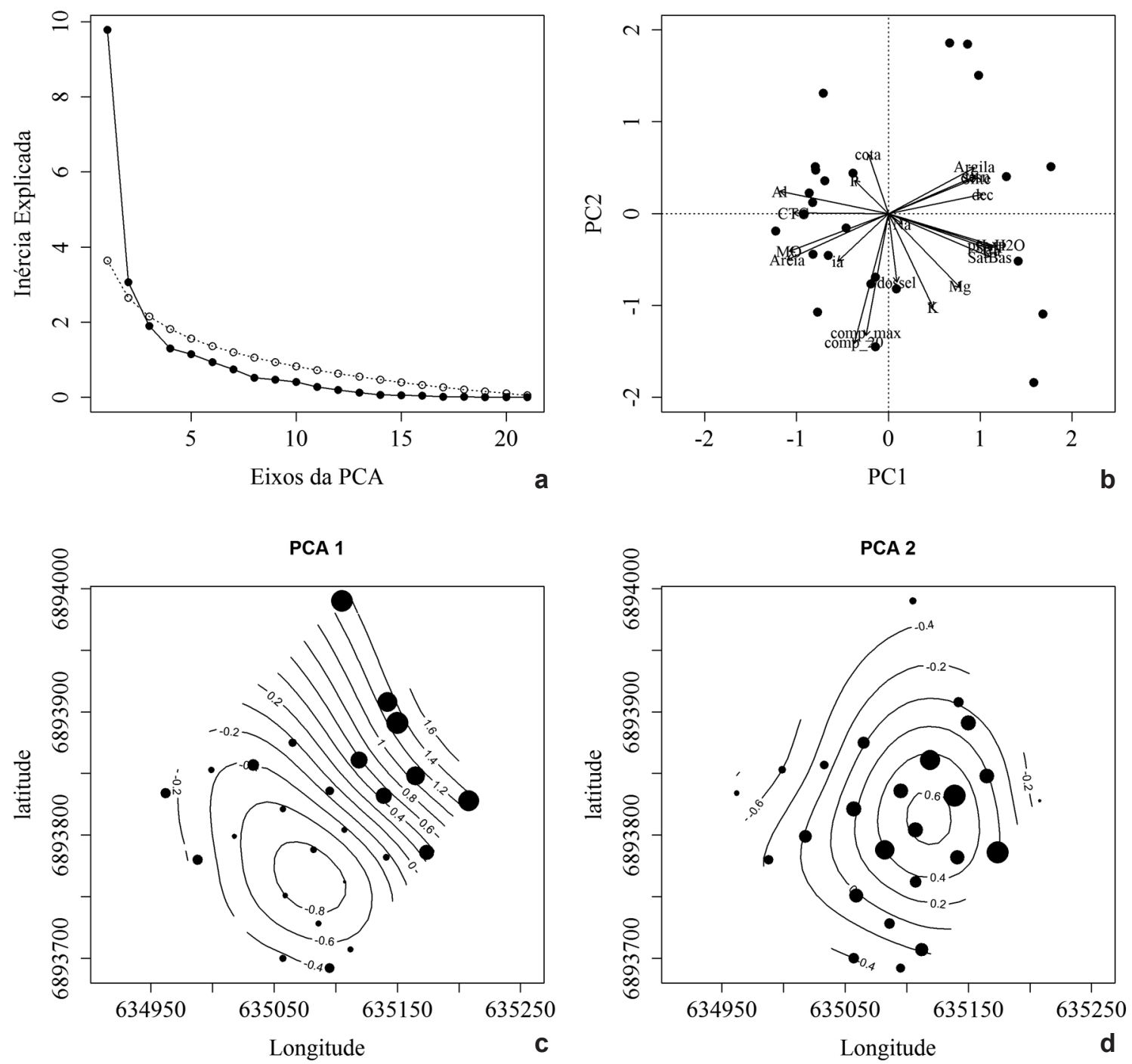

Figura 1 - Scree plot (a) para o resultado da ordenação produzida a partir da Análise de Componentes Principais das variáveis ambientais (b) em uma Floresta Nebular no sul do Brasil. Espacialização dos eixos 1 (c) e 2 (d) da PCA. Al = teor de alumínio; $\mathrm{CTC}=$ capacidade de troca cationica; $\mathrm{P}=$ teor de fósforo; $\operatorname{cota}=$ cota altitudinal; $\mathrm{MO}=$ matéria orgânica; Areia = teor de areia; ia = impacto ambiental; $\mathrm{Na}=$ teor de sódio; comp_20 = resistência à penetração a $20 \mathrm{~cm}$ de profundidade no solo; comp_max = resistência do valor máximo no perfil; dossel = cobertura do dossel; $\mathrm{K}=$ teor de potássio; $\mathrm{Mg}$ = teor de magnésio; $\mathrm{SatBas}=$ saturação por base; $\mathrm{pH} \mathrm{H2O=pH} \mathrm{em} \mathrm{água;} \mathrm{Ca}=$ cálcio; dec = declividade média; desn = desnível máximo; Silte = teor de silte; Argila = teor de argila. Na figura 1a, círculos fechados e linha contínua = Inércia observada; Círculo aberto e linha pontilhada = valores aleatórios esperados pela distribuição de Broken Stick. Nas figuras 1c e 1d, quanto maiores os tamanhos dos círculos fechados, maiores os valores dos scores das parcelas ao longo dos eixos 1 (1c) e 2 (1d) da PCA.

Figure 1 - Scree plot (a) for the results of ordination constructed through a Principal Component Analysis (PCA) of environmental variables (b), in a Cloud Forest in the South of Brazil. Spatialization of PCA Axis 1 (c) e 2 (d). Al = aluminum content; CTC= cation exchange capacity; $\mathrm{P}=$ phosphorus; $\operatorname{cota}=$ altitudinal quota; $\mathrm{MO}=$ organic matter; Areia $=$ sand content $; \mathrm{ia}=$ environmental impact; $\mathrm{Na}$ = sodium content; comp_20 = soil resistance to penetration at $20 \mathrm{~cm}$ deep; comp_max $=$ maximum resistance in soil profile; dossel $=$ canopy cover; $\mathrm{K}$ = potassium content; $\mathrm{Mg}$ = magnesium content; $\mathrm{SatBas}=$ base saturation; $\mathrm{pH} \mathrm{H} 2 \mathrm{O}=\mathrm{pH}$ in water; $\mathrm{Ca}=\mathrm{calcium}$; dec $=$ average slope desn = elevation gap; Silte = silt content; Argila = clay content. In figure 1a, closed circles and solid line $=$ Observed inertia; open circles and dotted line $=$ random values expected according to Broken Stick distribution. In figure 1c and 1d, the larger the size of the closed circle, the larger the values of plot scores along axis 1 (1c) and 2 (1d) of PCA. 
do solo (Fig. 1b). As parcelas ordenadas a esquerda situaram-se em locais com solos mais ácidos, menos férteis, mais arenosos e com topografia mais plana. Em outro extremo do gradiente, situaram-se as parcelas que apresentam solos menos ácidos, de maior fertilidade, mais argilosos e com topografia mais declivosa. No eixo 2, observa-se que as parcelas ordenadas na parte superior do gráfico apresentam solos menos compactados, com menores teores de $\mathrm{K} \mathrm{e} \mathrm{Mg,} \mathrm{com} \mathrm{cotas} \mathrm{altitudinais} \mathrm{mais} \mathrm{elevadas} \mathrm{e} \mathrm{com}$ dossel mais aberto; e na parte inferior situaram-se as parcelas com solos mais compactos, com maiores teores de $\mathrm{K}$ e $\mathrm{Mg}$, com menores cotas altitudinais e com dossel mais fechado. A espacialização dos valores dos scores das parcelas ao longo dos eixos 1 e 2 da PCA indica que ambos gradientes ambientais identificados ocorrem de forma espacialmente estruturada (Fig. 1c,d).
Atributos funcionais

As espécies que apresentaram os maiores valores observados para os atributos foram Ilex paraguariensis A.St.-Hil. $\left(\mathrm{AF}=19,59 \mathrm{~cm}^{2}\right)$, para a área foliar, Myrceugenia oxysepala (Burret) D.Legrand \& Kausel $\left(\mathrm{AFE}=132,69 \mathrm{~cm}^{2} \cdot \mathrm{g}^{-1}\right)$, para área foliar específica, Ilex microdonta Reissek (DBM $\left.=0,673 \mathrm{~g} \cdot \mathrm{cm}^{-3}\right)$, para densidade básica da madeira, e Myrsine coriacea (Sw.) R. Br. ex Roem. \& Schult. $(\mathrm{HMP}=20 \mathrm{~m})$, para altura máxima potencial. Já as espécies que apresentaram os menores valores observados foram Myrceugenia euosma (O.Berg) D.Legrand $\left(\mathrm{AF}=1,48 \mathrm{~cm}^{2}\right.$ e HMP $\left.=7 \mathrm{~m}\right)$, para área foliar e altura máxima potencial, Araucaria angustifolia (Bertol.) Kuntze (AFE $\left.=78,77 \mathrm{~cm}^{2} . \mathrm{g}^{-1}\right)$, para área foliar específica, e Cinnamomum amoenum (Nees \& Mart.) Kosterm. (DBM $=0,402 \mathrm{~g} \cdot \mathrm{cm}^{-3}$ ), para densidade básica da madeira (Tab. 1). Houve

Tabela 1 - Atributos funcionais das 19 espécies arbóreas mais abundantes amostradas em uma Floresta Nebular no sul do Brasil. Table 1 - Functional traits of the 19 most abundant species sampled in a Cloud Forest in the South of Brazil.

\begin{tabular}{|c|c|c|c|c|c|c|}
\hline \multirow{2}{*}{ Espécies } & \multicolumn{6}{|c|}{ Atributos } \\
\hline & $\mathbf{A F}$ & AFE & DBM & HMP & Dec & SD \\
\hline Araucaria angustifolia (Bertol.) Kuntze & $1,88(0,54)$ & 78,79 & $0,54(0,15)$ & 17 & $\mathrm{P}$ & Zoo \\
\hline Cinnamomum атоепит (Nees \& Mart.) Kosterm. & $6,59(3,55)$ & 83,48 & $0,40(0,06)$ & 15 & $\mathrm{P}$ & Zoo \\
\hline Drimys angustifolia Miers & $8,05(2,35)$ & 113,91 & $0,42(0,08)$ & 8 & $\mathrm{P}$ & Zoo \\
\hline Ilex microdonta Reissek & $8,87(2,52)$ & 86,01 & $0,67(0,25)$ & 17 & $\mathrm{P}$ & Zoo \\
\hline Ilex paraguariensis A.St.-Hil. & $19,59(6,97)$ & 106,48 & $0,46(0,04)$ & 15 & $\mathrm{P}$ & Zoo \\
\hline Maytenus boaria Molina & $5,07(2,67)$ & 98,17 & $0,60(0,06)$ & 15 & $\mathrm{P}$ & Zoo \\
\hline Myrceugenia euosma (O.Berg) D.Legrand & $1,48(0,56)$ & 104,39 & $0,56(0,05)$ & 7 & $\mathrm{P}$ & Zoo \\
\hline Myrceugenia glaucescens (Cambess.) D.Legrand \& Kausel & $3,97(1,68)$ & 106,08 & $0,60(0,09)$ & 11 & $\mathrm{P}$ & Zoo \\
\hline Myrceugenia miersiana (Gardner) D.Legrand \& Kausel & $6,97(2,93)$ & 127,68 & $0,50(0,05)$ & 8 & $\mathrm{SD}$ & Zoo \\
\hline Myrceugenia myrcioides (Cambess.) O.Berg & $15,86(5,67)$ & 111,76 & $0,55(0,04)$ & 12 & SD & Zoo \\
\hline Myrceugenia ovata (Hook. \& Arn.) O.Berg & $1,59(0,46)$ & 118,26 & $0,57(0,12)$ & 11 & $\mathrm{P}$ & Zoo \\
\hline Myrceugenia oxysepala (Burret) D.Legrand \& Kausel & $5,49(1,82)$ & 132,69 & $0,57(0,02)$ & 11 & $\mathrm{P}$ & Zoo \\
\hline Myrrhinium atropurpureum Schott & $8,46(3,11)$ & 81,77 & $0,67(0,13)$ & 12 & SD & Zoo \\
\hline Myrsine coriacea (Sw.) R. Br. ex Roem. \& Schult. & $8,68(3,01)$ & 94,57 & $0,55(0,04)$ & 20 & $\mathrm{P}$ & Zoo \\
\hline Ocotea pulchella Mart. & $5,23(1,61)$ & 107,89 & $0,52(0,06)$ & 18 & $\mathrm{P}$ & Zoo \\
\hline Prunus myrtifolia (L.) Urb. & $16,13(6,24)$ & 84,82 & $0,65(0,04)$ & 15 & $\mathrm{P}$ & Zoo \\
\hline Schinus polygamus (Cav.) Cabrera & $3,67(1,13)$ & 99,63 & $0,62(0,08)$ & 14 & $\mathrm{SD}$ & Zoo \\
\hline Symplocos tetrandra Mart. & $9,57(3,46)$ & 116,46 & $0,49(0,13)$ & 15 & $\mathrm{P}$ & Zoo \\
\hline Weinmannia paulliniifolia Pohl ex Ser. & $10,02(3,91)$ & 108,13 & $0,50(0,04)$ & 16 & $\mathrm{P}$ & Ane \\
\hline Média & 7,75 & 103,21 & 0,550 & 13,53 & - & - \\
\hline
\end{tabular}

AF: área foliar $\left(\mathrm{cm}^{2}\right)$; AFE: área foliar específica $\left(\mathrm{cm}^{2} \cdot \mathrm{g}^{-1}\right)$; DBM: densidade básica da madeira $\left(\mathrm{g} \cdot \mathrm{cm}^{-3}\right)$; HMP: altura máxima potencial $(\mathrm{m})$; Dec: deciduidade, sendo P = perenes e SD = semidecíduas; SD: síndrome de dispersão, sendo Zoo = zoocórica e Ane = anemocórica. Valores entres parênteses representam o desvio padrão do atributo avaliado. 
predomínio de espécies perenes e com síndrome de dispersão zoocórica. As únicas espécies que foram classificadas como semi-decíduas foram Myrceugenia miersiana (Gardner) D.Legrand \& Kausel, Myrcengenia myrcioides (Cambess.) O.Berg, Myrrhinium atropurpureum Schott e Schinus polygamus (Cav.) Cabrera, e a única anemocórica foi Weinmannia paulliniifolia Pohl ex Ser.

\section{Atributos funcionais $x$ gradientes} ambientais

Todos os modelos ajustados entre os aspectos funcionais (atributos e diversidade) e os gradientes ambientais apresentaram resíduos com distribuição normal, homocedasticidade e sem dependência espacial, indicando a adequabilidade dos modelos para interpretações. Observa-se que apenas o gradiente ambiental sintetizado pelo eixo 1 da PCA apresentou influência significativa sobre os atributos funcionais e diversidade funcional (Tab. 2). A PCA1 apresentou relação positiva significativa com área foliar e o índice de RaoQ e relação negativa significativa com a densidade básica da madeira. Assim, observou-se que nos locais de maior fertilidade, mais declivosos e argilosos são observados maiores valores de diversidade funcional e árvores com maiores áreas foliares e com madeiras menos densas. Já nos locais menos férteis, com solos mais ácidos, menos declivosos e mais arenosos, observou-se menor diversidade funcional e árvores com menores valores de área foliar e madeira mais densa.

Tabela 2 - Modelos lineares ajustados para avaliação da influência dos gradientes ambientais identificados (PCA1 e PCA2) sobre os atributos $(\mathrm{AF}=$ área foliar; $\mathrm{AFE}=$ área foliar específica; $\mathrm{DBM}=$ densidade básica da madeira; $\mathrm{HMP}=$ altura máxima potencial) e diversidade funcional (RaoQ) do componente arbóreo em uma Floresta Nebular no sul do Brasil. Table 2 - Linear Models fitted for the evaluation of the influence of identified environmental gradients (PCA1 and PCA2) on functional traits $(\mathrm{AF}=$ leaf area; $\mathrm{AFE}=$ specific leaf area; $\mathrm{DBM}=$ basic wood density; $\mathrm{HMP}=$ maximum potential height $)$ and functional diversity (RaoQ) of the tree component in a Cloud Forest in the South of Brazil.

\begin{tabular}{lcccc}
\hline & PCA1 & PCA2 & $\boldsymbol{p}$ & $\mathbf{R}^{2}$ \\
\hline AF & $0,8823^{*}$ & 0,2951 & 0,0006 & 0,4893 \\
AFE & 0,3086 & 0,6737 & 0,7779 & 0,0226 \\
DBM & $-0,0236^{*}$ & $-0,0076$ & 0,0003 & 0,4818 \\
HMP & 0,3217 & 0,2605 & 0,1580 & 0,1544 \\
RaoQ & $0,0092^{*}$ & 0,0032 & 0,0001 & 0,5229 \\
\hline
\end{tabular}

* Coeficientes angulares das variáveis independentes $\operatorname{com} p<0,001 . p=$ significância do modelo. $\mathrm{R}^{2}=$ ajuste linear do modelo.

\section{Discussão}

Os resultados encontrados indicaram que os gradientes ambientais identificados ocorreram de forma espacialmente estruturada, reforçando as observações de Marcon et al. (2014), que demonstraram a influência do relevo como um fator determinante das condições edáficas na área. Além disso, apesar do gradiente de fertilidade encontrado, é importante destacar, conforme já relatado por Marcon et al. (2014), que os solos da área de estudo apresentaram baixa fertilidade natural, sendo este um padrão comumente observado em outras áreas de elevada altitude (Falkenberg 2003; Homeier et al. 2010).

Estas condições de baixa fertilidade natural, juntamente com as características climáticas típicas de áreas nebulares de maior altitude, contribuem para a limitação do desenvolvimento de espécies arbóreas, refletindo, assim, nos atributos funcionais observados para o componente arbóreo. Quando comparado com outras florestas na região, situadas em pisos altitudinais inferiores e sem a influência de nuvens (e.g., Missio et al. 2017), o componente arbóreo dessas florestas se destacou pelo fato de ser, predominantemente, espécies perenifólias e com reduzida área foliar, sugerindo, assim, a existência de variações em escala regional, em repostas ao gradiente altitudinal, como também demonstrado por Foster (2001).

Quando analisada a relação entre a variação ambiental e os atributos funcionais, observou-se que a fertilidade do solo, juntamente com as outras variáveis com maior correlação com o eixo 1 da 
PCA, como o $\mathrm{pH}$, textura do solo e declividade, influenciaram de forma significativa a diversidade funcional, a área foliar e a densidade da madeira, indicando o particionamento de habitats em escala local ( $<1 \mathrm{ha})$ por espécies com estratégias ecológicas distintas. Nos locais de maior fertilidade do solo, que são aqueles localizados nas áreas de maior declive e com solos mais argilosos, ocorreu o favorecimento de estratégias que maximizam a obtenção de recursos, como a baixa densidade da madeira, que está associada a um crescimento mais rápido (Read et al. 2014), e a maior área foliar, relacionada com uma maior capacidade de captura de luz (Cornelissen et al. 2003). Por outro lado, nas áreas de menor fertilidade do solo e maior acidez, que são aquelas localizados nas áreas mais planas e com solos mais arenosos, predominaram estratégias de conservação de recursos adquiridos, como uma maior densidade da madeira, que confere uma maior resistência a danos mecânicos às espécies arbóreas (Reich 2014), e menor área foliar, que em um ambiente alto montano, representa uma estratégia para reduzir a superfície foliar exposta a baixas temperaturas durante o inverno (Foster 2001). Esta dicotomia de estratégias ecológicas tem sido frequentemente relatada na literatura (Díaz et al. 2004; Lohbeck et al. 2015; Sterck et al. 2011), sendo que a disponibilidade de nutrientes dos solos é considerada um dos principais fatores condicionantes das espécies que se estabelecerão em um determinado local (Díaz et al. 2004). Ainda, a menor diversidade funcional observada nos locais de menor fertilidade sugere que as condições ambientais existentes nesta parte da floresta representem um filtro ambiental para o estabelecimento de espécies, refletindo, assim, em uma menor amplitude funcional.

Conclui-se que mesmo em florestas condicionadas a fortes filtros ambientais, como as florestas nebulares alto-montanas ocorrentes no sul do Brasil, podem existir variações dos atributos funcionais em escala local em função de gradientes edáficos. Os resultados do presente estudo indicaram que o espaço horizontal da floresta nebular avaliada foi particionado por espécies com diferentes estratégias ecológicas em função de variações edáficas e topográficas. Este padrão sugere que a organização de comunidades de espécies arbóreas em florestas nebulares seja condicionada por fatores ecológicos determinísticos, associados às variações ambientais existentes.

\section{Agradecimentos}

À FAPESC (Fundação de Amparo à Pesquisa e Inovação do estado de Santa Catarina), a concessão de bolsa de Mestrado à primeira autora do trabalho, e o apoio financeiro por meio do Edital PAP-UDESC. Ao CNPq, a concessão de bolsa de Produtividade para o segundo e o terceiro autores.

\section{Referências}

Apaza-Quevedo, A.; Lippok, D.; Hensen, I.; Schleuning, M. \& Both, S. 2015. Elevation, topography and edge effects drive functional composition of woody plant species in Tropical Montane Forests. Biotropica 47: 449-458.

Becker, A.; Körner, C.; Brun, J.; Guisan, A. \& Tappeiner, U. 2007. Ecological and land use studies along elevational gradients. Mountain Research and Development 27: 58-65.

Bjornstad, O.N. 2013. ncf: spatial nonparametric covariance functions. R package version 1.1-5. 2013. Disponível em <http://cran.r-project.org/ package $=$ ncf $>$. Acesso em 24 julho 2015 .

Botta-Dukát, Z. 2005. Rao's quadratic entropy as a measure of functional diversity based on multiple traits. Journal of vegetation science 16: 533-540.

Chave, J. 2005. Measuring wood density for tropical forest trees: a field manual for the CTFS sites. Laboratoire Evolution et Diversité Biologique, Toulouse. 7p.

Cornelissen, J.H.C.A.; Lavorel, S.B.; Garnier, E.B.; Díaz, S.C.; Buchmann, N.D.; Gurvich, D.E.C.; Reich, P.B.E.; Steege, H.F.; Morgan, H.D.G.; Van Der Heijden, A.M.G.A.; Pausas, J.G.H. \& Poorter, H.I. 2003. A handbook of protocols for standardised and easy measurement of plant functional traits worldwide. Australian Journal of Botany 51: 335380.

Díaz, S.; Hodgson, J.G.; Thompson, K.; Cabido, M.; Cornelissen, J.H.C.; Jalili, A.; MontserratMartí, G.; Grime, J.P.; Zarrinkamar, F.; Asri, Y.; Band, S.R.; Basconcelo, S.; Castro-Díez, P.; Funes, G.; Hamzehee, B.; Khoshnevi, M.; PérezHarguindeguy, N.; Pérez-Rontomé, M.C.; Shirvany, F.A.; Vendramini, F.; Yazdani, S.; Abbas-Azimi, R.; Bogaard, A.; Boustani, S.; Charles, M.; Dehghan, M.; Torres-Espuny, L.; Falczuk, V.; GuerreroCampo, J.; Hynd, A.; Jones, G.; Kowsary, E.; Kazemi-Saeed, F.; Maestro-Martínez, M.; RomoDíez, A.; Shaw, S.; Siavash, B.; Villar-Salvador, P. \& Zak, M.R. 2004. The plant traits that drive ecosystems: evidence from three continents. Journal of Vegetation Science 15: 295-304.

Falkenberg, D. 2003. Matinhas nebulares e vegetação rupícola dos Aparados da Serra Geral (SC/RS), sul do Brasil. Tese de Doutorado. Universidade Estadual de Campinas, Campinas. 558p. 
Falkenberg, D.B. \& Voltolini, J.C. 1995. The montane cloud forest in southern Brazil. In: Hamilton, L.S.; Juvik, J.O. \& Scatena, F.N. (eds.). Tropical montane cloud forests. Springer-Verlarg, New York. Pp. 138-149.

Fernandes, M.F. \& Queiroz, F.P. 2015. Floristic surveys of Restinga Forests in southern Bahia, Brazil, reveal the effects of geography on community composition. Rodriguésia 66: 51-73.

Foster, P. 2001. The potential negative impacts of global climate change on tropical montane cloud forests. Earth-Science Reviews 55: 73-106.

Garnier, E. \& Navas, M. 2012. A trait-based approach to comparative functional plant ecology: concepts, methods and applications for agroecology. A review. Agronomy for Sustainable Development, Springer Verlag/EDP Sciences/INRA 32: 365-399.

Higuchi, P.; Silva, A.C.; Almeida, J.A.; Bortoluzzi, R.L.C.; Mantovani, A.; Ferreira, T.S.; Souza, S.T.; Gomes, J.P. \& Silva, K.M. 2013. Florística e estrutura do componente arbóreo e análise ambiental de um fragmento de Floresta Ombrófila Mista Alto-montana no município de Painel, SC. Ciência Florestal 23: 153-164.

Homeier, J.; Breckle, S.W.; Günter, S.; Rollenbeck, R.T. \& Leuschner, C. 2010. Tree diversity, forest structure and productivity along altitudinal and topographical gradients in a species rich ecuadorian Montane Rain Forest. Biotropica 42: 140-148.

IBGE. 2012. Manual técnico da vegetação brasileira. Fundação Instituto Brasileiro de Geografia e Estatística, Rio de Janeiro. 323p.

Kitayama, K. \& Aiba, S.I. 2002. Ecosystem structure and productivity of tropical rain forests along altitudinal gradients with contrasting soil phosphorus pools on Mount Kinabalu, Borneo. Journal of Ecology 90: 37-51.

Kraft, N.J.B.; Valencia, R. \& Ackerly, D.D. 2008. Functional traits and niche-based tree community assembly in na Amazonian Forest. Science 322: 580-582.

Laliberté, E. \& Shipley, B. 2014. FD: measuring functional diversity from multiple traits, and other tools for functional ecology. R package version 1.0-12. Disponível em <http://www.elaliberte.info/ publications $>$. Acesso em 24 julho 2015.

Leigh, E.G. 1975. Structure and climate in tropical rain forest. Annual Review of Ecology and Systematics 6: 67-86.

Lohbeck, M.; Lebrija-Trejos, E.; Martínez-Ramos, M.; Meave, J.A.; Poorter, L. \& Bongers, F. 2015. Functional trait strategies of trees in dry and wet tropical forests are similar but differ in their consequences for succession. Plos One 10: 1-15.

Lorenzi, H. 2002. Árvores Brasileiras: manual de identificação e cultivo de plantas arbóreas nativas do Brasil. Vol. 2. 2a . ed. Instituto Plantarum, Nova Odessa. 384p.
Lorenzi, H. 2008. Árvores Brasileiras: manual de identificação e cultivo de plantas arbóreas nativas do Brasil. Vol. 1. 5a. ed. Instituto Plantarum, Nova Odessa. 384p.

Lorenzi, H. 2009. Árvores brasileiras: manual de identificação e cultivo de plantas arbóreas nativas do Brasil. Vol. 3. Instituto Plantarum, Nova Odessa. $384 \mathrm{p}$.

Maçaneiro, J.P.; Oliveira, L.Z.; Seubert, R.C.; Eisenlohr, P.V. \& Schorn, L.A. 2016. More than environmental control at local scales: do spatial processes play an important role in floristic variation in subtropical forests? Acta Botanica Brasilica. Disponível em <https://dx.doi.org/10.1590/010233062015abb0294>. Acesso em 12 abril 2016.

Magnago, L.F.S.; Edwards, D.P.; Edwards, F.A.; Magrach, A.; Martins, S.V. \& Laurance, W.F. 2014. Functional attributes change but functional richness is unchanged after fragmentation of Brazilian Atlantic forests. Journal of Ecology 102: 475-485.

Marcon, A.K.; Silva, A.C.; Higuchi, P.; Ferreira, T.S.; Missio, F.F. \& Salami, B. 2014. Variação florísticoestrutural em resposta à heterogeneidade ambiental em uma floresta nebular em Ububici, Planalto Catarinense. Scientia Forestalis 42: 439-450.

Martins, S.V.; Colleti Júnior, C.; Rodrigues, R.R. \& Gandolfi, S. 2004. Colonization of gaps produced by death of bamboo clumps in a semideciduous mesophytic forest in south-eastern Brazil. Plant Ecology 172: 121-131.

Martínez, M.L.; Pérez-Maqueo, O.; Vázquez, G.; CastilloCampos, G.; García-Franco, J.; Mehltreter, K.; Equihua, M. \& Landgrave, R. 2009. Effects of land use change on biodiversity and ecosystem services in tropical montane cloud forests of Mexico. Forest Ecology and Management 258: 1856-1863.

Missio, F.F.; Silva, A.C.; Higuchi, P.; Longhi, S.J.; Brand, M.A.; Rios, P.D.; Dalla Rosa, A.; Buzzi Junior, F.; Bento, M.A.; Gonçalves, D.A.; Loebens, R. \& Pscheidt, F. 2017 Atributos funcionais de espécies arbóreas em um fragmento de Floresta Ombrófila Mista em Lages, SC. Ciência Florestal 27: 215-224.

Moro, M.F.; Macedo, M.B.; Moura-Fé, M.M.; Castro, A.S.F. \& Costa, R.C. 2015. Vegetation, phytoecological regions and landscape diversity in Ceará state, northeastern Brazil. Rodriguésia 66: 717-743.

Negrini, M.; Aguiar, M.D.; Vieira, C.T.; Silva, A.C. \& Higuchi, P. 2012. Dispersão, distribuição espacial e estratificação vertical da comunidade arbórea em um fragmento florestal no Planalto Catarinense. Revista Árvore 36: 919-930.

Oksanen, J.; Blanchet, G.; Kindt, R.; Legendre, P.; Minchin, R.P.; O’hara, R.B.; Simpson, L.G.; Solymos, P.; Stevens, H.H.M. \& Wagner, H. 2015. Vegan: community ecology package. R package version 2.3-1. Disponível em <http://CRAN.R-project.org/ package= $=$ vegan $>$. Acesso em 24 julho 2015 . 
Oliveira-Filho, A.T. \& Fontes, M.A.L. 2000. Patterns of floristic differentiation among atlantic forests in southeastern Brazil and the influence of climate1. Biotropica 32: 793-810.

Pakeman, R.J. \& Quested, H.M. 2007. Sampling plant functional traits: what proportion of the species need to be measured? Applied Vegetation Science 10: 91-96.

Pandolfo, C.; Braga, H.J.; Silva Júnior, V.P.; Massignan, A.M.; Pereira, E.S.; Thomé, V.M.R. \& Valci, F.V. 2002. Atlas climatológico do estado de Santa Catarina. Disponível em $<$ http://ciram.epagri. sc.gov.br/atlas_climatologico/>. Acesso em 24 outubro 2015.

Pérez-Harguindeguy, N.; Díaz, S.; Garnier, E.; Lavorel, S.; Poorter, H.; Jaureguiberry, P.; Bret-Harte, M.S.; Cornwell, W.K.; Craine, J.M.; Gurvich, D.E.; Urcelay, C.; Veneklaas, E.J.; Reich, P.B.; Poorter, L.; Wright, I.J.; Ray, P.; Enrico, L.; Pausas, J.G.; De Vos, A.C.; Buchmann, N.; Funes, G.; Quétier, F.; Hodgson, J.G.; Thompson, K.; Morgan, H.D.; Ter Steege, H.; Van Der Heijden, M.G.A.; Sack, L.; Blonder, B.; Poschlod, P.; Vaieretti, M.V.; Conti, G.; Staver, A.C.; Aquino, S. \& Cornelissen, J.H.C. 2013. New handbook for standardised measurement of plant functional traits worldwide. Australian Journal of Botany 61: 167-234.

R Development Core Team. 2015. R: a language and environment for statistical computing. $\mathrm{R}$ Foundation for Statistical Computing, Vienna, Austria. Disponível em <http:/www.r-project.org $>$. Acesso em 24 julho 2015.

Rao, C.R. 1982. Diversity and dissimilarity coefficients: a unified approach. Theoretical Population Biology 21: 24-43.

Rasband, W.S. 2007. ImageJ. Bethesda, USA: National Institute of Health. Disponível em $<$ http://imagej. nih.gov/ij/>. Acesso em 24 outubro 2014.

Read, Q.D.; Moorhead, L.C.; Swenson, N.G.; Bailey, J.K. \& Sanders, N.J. 2014. Convergent effects of elevation on functional leaf traits within and among species. Functional Ecology 28: 37-45.

Reich, P.B. 2014. The world-wide 'fast-slow' plant economics spectrum: a traits manifesto. Journal of Ecology 102: 275-301.

Rosado, B.H.P.; Joly, C.A.; Burgess, S.S.O.; Oliveira, R.S. \& Aidar, M.P. 2016. Changes in plant functional traits and water use in Atlantic rainforest: evidence of conservative water use in spatio-temporal scales. Trees 30: 47-61.

Safford, H.D. 1999. Brazilian Paramos I. An introduction to the physical environment and vegetation of the campos de altitude. Journal of Biogeography 26: 693-712.

Safford, H.D. 2007. Brazilian Paramos IV: Phytogeography of the campos de altitude. Journal of Biogeography 34: 1701-1722.

Scheer, M.B. \& Mocochinski, A.Y. 2009. Florística vascular da Floresta Ombrófila Densa Altomontana de quatro serras no Paraná. Biota Neotropica 9: 51-69.

Scheer, M.B.; Curcio, G.R. \& Roderjan, C.V. 2011a. Funcionalidades ambientais de solos altomontanos na Serra da Igreja, Paraná. Revista Brasileira de Ciência do Solo 35: 1113-1126.

Scheer, M.B.; Mocochinski, A.Y. \& Roderjan, C.V. 2011b. Estrutura arbórea da Floresta Ombrófila Densa Altomontana de serras do Sul do Brasil. Acta Botanica Brasilica 25: 735-750.

Souza, K.; Souza, C.C.; Rosa, M.G.; Cruz, A.P.; Lima, C.L.; Silva, J.O.; Lazzarin, L.C.; Loebens, R.; Dias, R.A.R.; Silva, A.C.; Higuchi, P. \& Schimalski, M.B. 2015. Estrutura e estratégias de dispersão do componente arbóreo de uma floresta subtropical ao longo de uma topossequência no Alto-Uruguai. Scientia Forestalis 43: 321-332.

Sterck, F.; Markesteijn, L.; Schieving, F. \& Poorter, L. 2011. Functional traits determine trade-offs and niches in a tropical forest community. Proceedings of the National Academy of Sciences 108: 20627-20632.

Torres, R.B; Martins, F.R.; \& Kinoshita, L.S. 1997. Climate, soil and tree flora relationships in forests in the state of São Paulo, southeastern Brasil. Brazilian Journal of Botany 20: 41-49.

Van Der Pijl, L. 1982. Principles of dispersal in higher plants. Springer-Verlarg, Berlin. 161p.

Violle, C.; Navas, M.; Vile, D.; Kazakou, E.; Fortunel, C.; Hummel, I. \& Garnier, E. 2007. Let the concept of trait be functional! Oikos 116: 882892.

Violle, C. \& Jiang, L. 2009. Towards a trait-based quantification of species niche. Journal of Plant Ecology 2: 87-93. 